\title{
Confecção de sensores de vidro poroso sinterizado para medida do potencial mátrico
}

\section{(Processing of porous glass sensors for matric potential measurement)}

\author{
O. B. G. Assis, J. D. C. Pessoa, A. G. Calbo \\ Embrapa Instrumentação, Rua XV de novembro, 1452, C.P. 741, S. Carlos, SP 13560-970 \\ odilio.assis@gmail.com
}

\begin{abstract}
Resumo
Sensores de contato apropriados para a avaliação do potencial hidrostático do apoplasma ou do potencial mátrico foram processados por técnicas cerâmicas. Os sensores propostos são formados por uma interface porosa e um capilar de vidro preenchido com água e um fluido imiscível. A sucção da água através da interface causa a deformação elástica de um dos fluidos e o conseqüente deslocamento do menisco, que é a base da medida. A interface porosa foi produzida por sinterização sob ação de chama a partir de pós de vidro pelo princípio de preenchimento (filler principle). Empregou-se mistura de pó de vidro e sal ( $\mathrm{NaCl}$ ) na proporção de 70 $30 \%$ e com dimensões médias de $17 \mu \mathrm{m}$. Testes de absorção de água destilada foram realizados indicando uma maior capilaridade nos sensores com a presença da matriz porosa, refletindo no aumento da velocidade do deslocamento do menisco ao longo do capilar. Palavras-chave: vidro poroso, sinterização viscosa, sensores, potencial mátrico.
\end{abstract}

\begin{abstract}
Contact probes suitable for hydraulic apoplasm or matric potential determination were processed by ceramic technique. The proposed probe consisted of a porous interface and a glass capillary filled with water and an immiscible fluid. The water conductivity through the interface generates elastic deformation of the fluid and consequent the meniscus dislocation, taken as measurement reference. The porous interface was processed by sintering under flame heating of glass powder following the "filler principle". Powder mixtures of glass and salt ( $\mathrm{NaCl}$ in $70-30 \%$ and average particle size of $17 \mu \mathrm{m}$ was used. Adsorption measurements carried out in distilled water show an increasing in capillarity effect in the sensors with the porous bridge, reflecting in an increasing over the meniscus speed movement as measured along the capillary.
\end{abstract}

Keywords: porous glass, viscous sintering, sensors, matric potential.

\section{INTRODUÇÃO}

A compreensão plena dos fenômenos que regem a sinterização de vidros ocorreu no período de 1976 a 1985 com a série de trabalhos publicados por Scherer [1-3], nos quais os principais princípios da densificação por sinterização viscosa foram modelados e discutidos de forma tutorial e prática. Nestes trabalhos, os mecanismos que regem a sinterização de estruturas amorfas, ficaram melhor esclarecidos permitindo o estabelecimento de parâmetros experimentais que possibilitaram a confecção de múltiplos diapositivos vítreos porosos com diversos graus de densidade e complexidade no formato. Scherer, por sua vez, teve por base os modelos apresentados por Frenkel [4] e por Kuczynski [5], nos quais ficaram estabelecidos a necessidade de uma força inicial (tensão superficial) para desencadear o movimento atômico e, conseqüentemente, para que o processo de sinterização seja iniciado. $\mathrm{Na}$ definição clássica de fluxo, segundo Frenkel [4], o sistema é regido por uma equação de estado simples do tipo:

$$
\overrightarrow{\mathrm{F}}=\eta \dot{\varepsilon}
$$

sendo: $\overrightarrow{\mathrm{F}}=$ força atuante, $\dot{\varepsilon}=$ taxa de cisalhamento e $\mathrm{h}=$ coeficiente de viscosidade

Ou seja, ao aquecermos o sistema o mecanismo de densificação terá inicio pelo movimento, isolado ou contínuo, de átomos na direção das vacâncias vizinhas (cisalhamento), preferencialmente na direção de uma força atuante (tensão superficial). Temos assim um aspecto diferencial e determinante na sinterização de vidros: o fluxo viscoso. A formação de pescoço e a subseqüente eliminação de poros (densificação) não dependem somente da temperatura e do tempo, mas predominantemente do escoamento contínuo de matéria, o que pode levar a uma sinterização muito mais rápida do que as estabelecidas para a maioria dos óxidos cerâmicos. Assim, a confecção de um vidro denso ou poroso a partir da junção de partículas isoladas é inicialmente regida pelas tensões estabelecidas pelo contato entre as partículas, que com o acréscimo da energia (temperatura) dá inicio ao processo de sinterização propriamente dito, com 
o deslocamento de massa preferencialmente na direção de maior tensão. Com o pleno entendimento desse mecanismo, o processamento de matrizes vítreas via sinterização permitiu o desenvolvimento de uma série de dispositivos que atualmente já se encontram em uso corrente nas mais distintas áreas. Podemos citar, por exemplo, sistemas de purificação de ar [6], sensores para a avaliação da umidade ambiental [7], ou dispositivos de captura de gases gerados na combustão de motores a etanol [8] que fazem uso de vidro sinterizado em seus compartimentos principais.

$\mathrm{Na}$ indústria química temos detectores de vazamentos de pequenas quantidades de voláteis orgânicos tóxicos [9] ou matriz vítrea porosa como meio de interação em reatores fotocatalílicos para degradação de contaminantes orgânicos [10]. Estruturas com base em vidros porosos sinterizados também têm sido promissoras em uso médico, com os chamados "bioglass" confeccionados para aplicações como implantes biocompatíveis em substituição aos ossos ou articulações [11, 12], ou no emprego como suporte para a imobilização de enzimas e anticorpos para análises em sistemas de diagnóstico rápido do tipo ELISA [13, 14]. Cartuchos vítreos porosos também têm sido empregados como indicativos de qualidade de ar em sistema de respiração extracorpórea de uso hospitalar [15].

$\mathrm{Na}$ agricultura e meio ambiente, membranas vítreas sinterizadas foram avaliadas na remoção de agrotóxicos [16], na liberação de feromônios para controle da infestação de insetos [17] e na medida do potencial hídrico em solos [18].

A vantagem no uso de dispositivos confeccionados com vidro poroso sinterizado está em sua estabilidade química e dimensional associada a altas áreas superficial que podem ser obtidas nas matrizes porosas. Associam-se a estas características um aumento significativo da capilaridade e transparência o que permite o controle visual e uma limpeza mais eficiente proporcionando um reuso mais duradouro [19].

\section{Dispositivo para medida do potencial mátrico}

O potencial da água e seus componentes em plantas tais como turgescência, sucção, potencial osmótico do simplasma e do aploplasma são variáveis empregadas na caracterização de condições críticas de déficit hídrico [20]. Em condições normais, um decréscimo da ordem de $0,1 \mathrm{MPa}$ no potencial de água na raiz de uma planta é suficiente para comprometer o crescimento celular e afetar a síntese de proteínas e demais atividades enzimáticas [21].

No Brasil, poucos estudos existem com respeito a relação entre o potencial mátrico em plantas e produtividade, em função principalmente da ausência de dispositivos sensíveis a este tipo de medida [22]. Na unidade da Embrapa Instrumentação em S. Carlos, SP, encontra-se em desenvolvimento uma base experimental para a medida da pressão interna em células vegetais baseado no sistema introduzido por Pessoa \& Calbo [23] adaptado à medida de turgescência (pressão intracelular), o que possibilita estimar valores relativos de deficiência hídrica em plantas. A base de medida neste equipamento é o registro do deslocamento de um menisco em uma sonda composta de um tubo capilar vítreo com diâmetro com bulbo entre $100 \mu \mathrm{m}$ e $200 \mu \mathrm{m}$ e ponta em cônica em torno de $7 \mu \mathrm{m}$, suficiente para penetrar em uma célula vegetal sem rompê-la. O capilar é conectado a uma câmara e ambos são preenchidos com um líquido com fator de compressibilidade $\mathrm{K}$ conhecido. O princípio de medida é o de balancear a turgescência da célula em oposição à pressão do volume preenchido. Detalhes experimentais da realização das medidas podem ser encontrados nas referencias $[24,25]$.

O objetivo do presente trabalho, em específico, é o de obter uma região porosa na extremidade do capilar (região que será introduzida ou estará em contato com o tecido vegetal), com o intuito de estabilizar o gradiente de pressão na interface e elevar a capilaridade, ao mesmo tempo reduzindo a fragilidade da ponta. Para a formação dessa "ponte" porosa de maior tortuosidade, optou-se pelo processamento de sinterização segundo a técnica de preenchimento (filler principle), a qual faz uso da mistura de partículas de vidro e de uma fase solúvel em água, não-sinterizável, que após o ciclo de consolidação térmica é removida resultando em uma matriz porosa. $\mathrm{O}$ uso da técnica de preenchimento para a obtenção de vidros sinterizados é um processo bem estabelecido e detalhadamente descrito por Siebers e co-autores [26] e tem sido empregada na confecção de elementos vítreos filtrantes com porosidade controlada [16].

\section{EXPERIMENTAL}

Vidro plano transparente de origem comercial de composição média $\mathrm{CaO}(10-15 \%) \quad \mathrm{Na}_{2} \mathrm{O}(12-15 \%) \mathrm{SiO}_{2}$ (71-75\%), (dados fornecidas pelo fabricante [27]), foi manualmente fragmentado em dimensões inferiores a 100 $\mu \mathrm{m}$ e misturados com $30 \%$ (em peso) de $\mathrm{NaCl}$ seguido de moagem em moinho de bolas de alumina por $2 \mathrm{~h}$. A função do sal é estabelecer uma fase intermediária, não sinterizável que possa ser facilmente removida após o processo de consolidação da fase vítrea. O pó após a moagem (vidro + sal) é o material de preenchimento da ponte porosa (região ao redor de $1 \mathrm{~mm}$ ) na extremidade aberta do capilar.

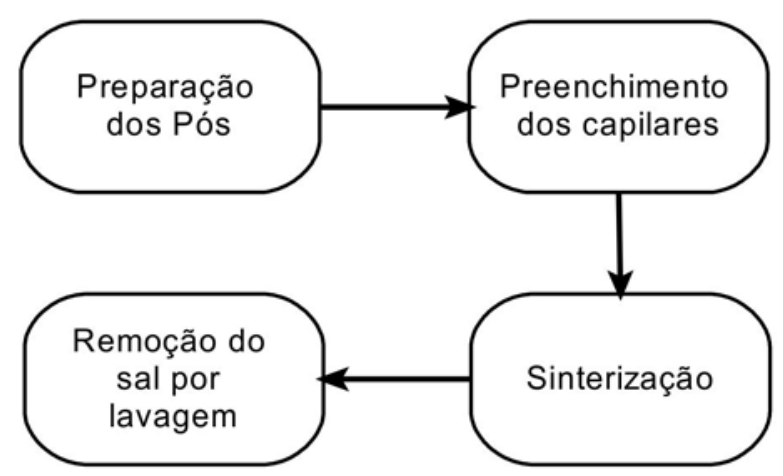

Figura 1: Seqüência básica do processamento do meio poroso pela técnica de preenchimento.

[Figure 1: Basic sequence of porous medium processing according to the filler principle technique.] 
Utilizou-se hematocapilares vítreos comerciais, sendo o pó manualmente compactado. A sinterização da região porosa se deu por passagens em chama de bico de Bunsen por breves intervalos de tempo. A remoção do solvente foi conduzida por intensiva passagem de água corrente através do capilar. A Fig. 1 apresenta a seqüência básica do processamento empregado (filler principle) [26].

A morfologia e a dimensão aproximada dos grãos foram avaliadas por microscopia eletrônica de varredura (Philips XL 30). Para a observação da região sinterizada, o sensor foi embutido em resina, seccionado transversalmente e revestido com ouro (Balzers Union Sputtering).

O sensor em sua configuração final fica assim composto por um capilar de vidro transparente e de uma parcela (interface) porosa. Quando saturada com água ou um fluido imiscível de compressibilidade (k), permite avaliar o potencial de sucção de fluído da amostra, tomada como igual à tensão de reação $(\mathrm{P})$ do corpo fluido, ou seja:

$$
\mathrm{dP}=-\frac{1}{\mathrm{k}} \frac{\mathrm{dV}}{V}
$$

sendo $\mathrm{V}$ o volume inicial do fluido deformado e $\mathrm{d}$ o operador diferencial. No capilar de simetria cilíndrica, a variação relativa de volume pode ser substituída pela variação relativa da posição do menisco fluido1/fluido2, Fig. 2. A faixa de valores mensurável com esta metodologia depende da quantidade de gases dissolvida nos fluidos, das dimensões dos poros e do volume disponível na interface porosa, do raio capilar (r) e da tensão superficial da água (s). Em uma avaliação preliminar [24]:

$$
\mathrm{P}=\frac{2 \cdot \sigma \cdot \cos \theta}{\mathrm{r}}
$$

admite-se $\cos \theta=1$ e o raio capilar próximo ao limite inferior para visualização no microscópio óptico: $1 \mathrm{~mm}$. Com este modelo e nestas condições a tensão máxima medida pelo

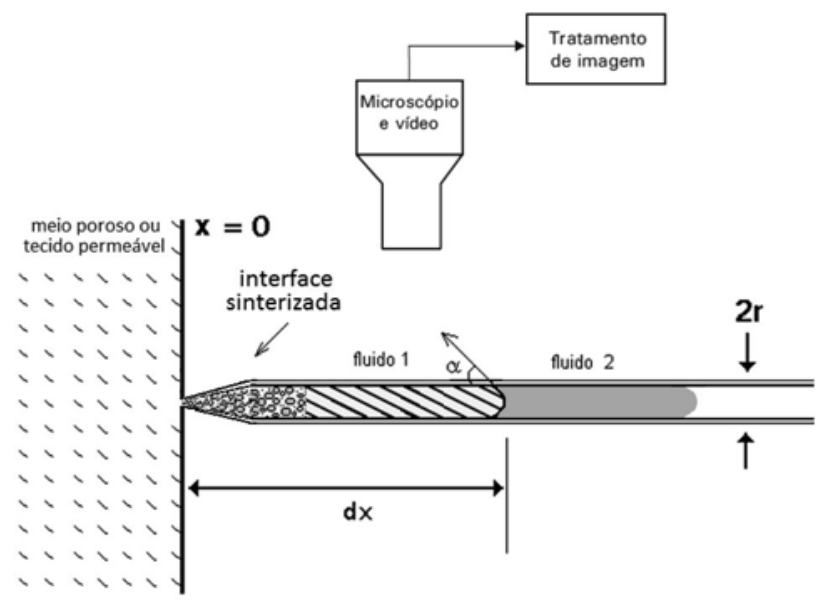

Figura 2: Princípio e disposição padrão do sensor de contato com região porosa sinterizada [21].

[Figure 2: Principle and standard arrangement of the contact sensor with porous sintered bridge [21].] sensor é da ordem de $1 \mathrm{MPa}$, limite adequado para muitas aplicações em física dos solos e fisiologia vegetal [23, 24].

\section{RESULTADOS E DISCUSSÃO}

Os parâmetros de moagem e fração de adição de sal foram escolhidos por serem aqueles que apresentaram os melhores resultados segundo estudos anteriormente conduzidos no processamento de matrizes de alta porosidade [16, 28]. Na Fig. 3a temos o aspecto microscópico da mistura (vidro + sal) obtida por moagem após $2 \mathrm{~h}$. Medidas quantitativas indicam uma larga distribuição de tamanho de grãos com média em valores próximos a $17 \mu \mathrm{m}$, conforme histograma apresentado na Fig. 3b.
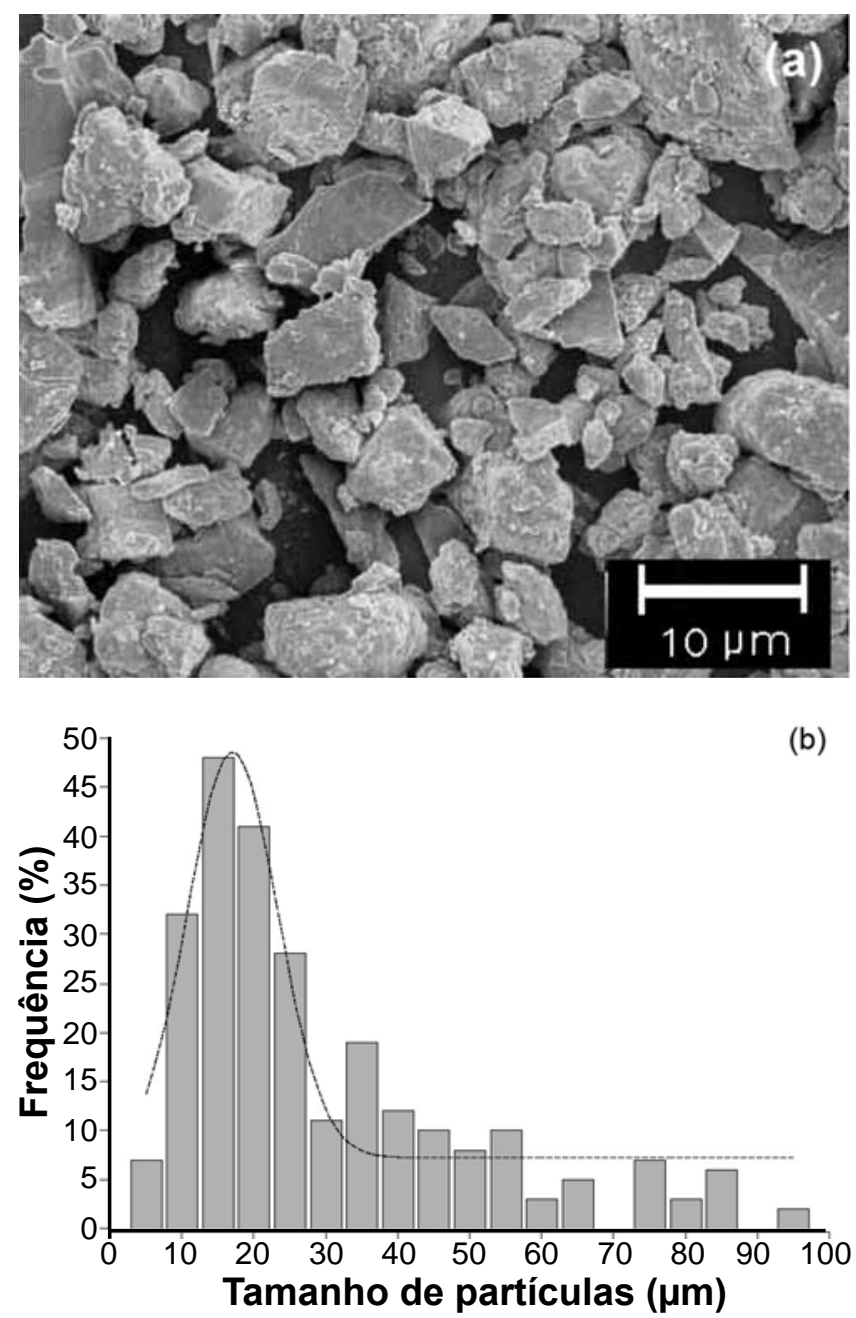

Figura 3: Aspecto microscópico após $2 \mathrm{~h}$ de moagem vidro + sal (a) e distribuição de tamanhos de partículas neste período (b).

[Figure 3: Microscopic aspect of glass + salt powder after $2 \mathrm{~h}$ ground (a) and the particle size distribution in this period (b).]

No processo adotado, devido ao curto período de aquecimento, a sinterização ocorre de forma incompleta com o estabelecimento inicial de pescoço mínimo entre as partículas, configurando contudo um corpo rígido. Independente das dimensões dos pós, a literatura apresenta temperaturas entre 600 a $800^{\circ} \mathrm{C}$ como ideais para que ocorra 
a plena sinterização de vidros sódio-silicatos [2, 29]. Ao atingir a temperatura de amolecimento, em torno de 536$696{ }^{\circ} \mathrm{C}$ [29], tem-se o inicio do processo de adesão entre as partículas. Nessas condições, os pescoços inicialmente estabelecidos apresentam raios de curvaturas menores aos comparados com a curvatura da superfície das partículas, o que resulta em uma pressão negativa em direção ao pescoço. Esse gradiente de pressão precipita o movimento viscoso para a região inter-partículas, com rápido deslocamento de massa e subseqüente coalescimento das partículas. Uma vez iniciado este fluxo, há pouco controle externo sobre a estrutura final resultante em função da velocidade do processo, o que no entanto pode ser interrompido pela súbita remoção da temperatura. Empregamos uma chama produzida por um bico de Bunsen como fonte térmica para o aquecimento da região do capilar preenchido como o pó. O procedimento compreendeu em três passagens pela zona térmica e sua instantânea retirada para rápido resfriamento. Como a temperatura da chama do bico varia de $300{ }^{\circ} \mathrm{C}$ a $1600^{\circ} \mathrm{C}$, dependendo de sua região (cor) [30], ocorre o inicio da sinterização garantindo a consolidação da fase vítrea. Como o processo é interrompido oportunamente (anterior a

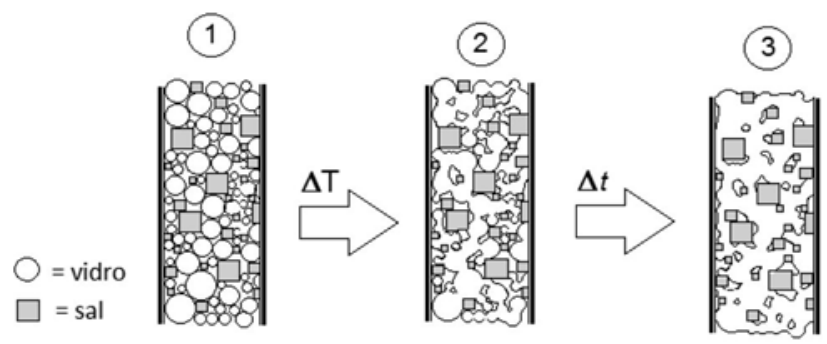

(4)

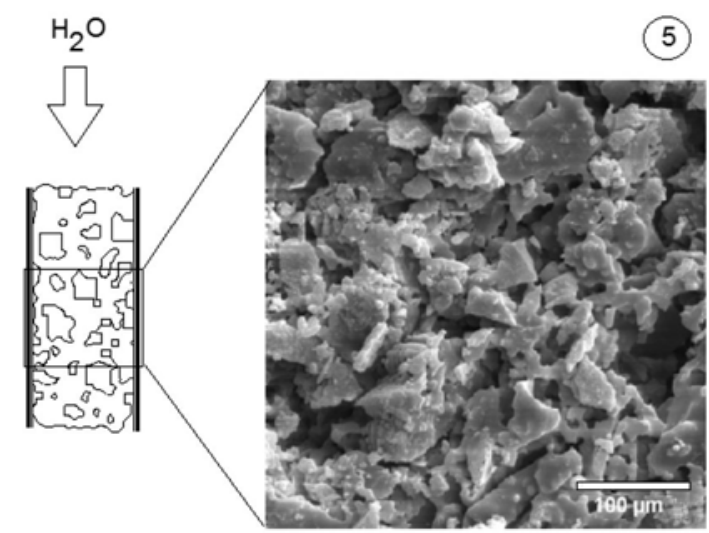

Figura 4: Esquema ideal de formação da região porosa segundo o "principio do preenchimento": em (1) temos a distribuição vidrosal compactado dentro do capilar. Com a ação da temperatura damos inicio à sinterização estabelecendo a consolidação sólida da fase vítrea (2) e (3). Com a remoção do sal por lixiviação em água corrente (4) estabelecemos a matriz porosa ao longo do capilar (5). [Figure 4: Ideal condition for porous medium formation according to the "filler principle": in (1) we have the glass-salt powder compacted into the capillary. With heating the sintering starts stablishing the vitreous solid phase consolidation (2) and (3). With the salt withdraw via lixiviation under running water (4) a porous matrix is formed (5).] um completo encapsulamento das partículas de sal), após a lixiviação da fase solúvel em água corrente estabelecemos uma matriz altamente porosa.

A Fig. 4 ilustra a seqüência ideal e apresenta o aspecto microscópio real da região porosa na extremidade do capilar.

Um aspecto importante a ser considerado na confecção e uso desses elementos está relacionado com o empacotamento final das partículas e com o formato dos poros, que tem influência no efeito de capilaridade dentro do sensor. Uma estrutura porosa composta de partículas/grãos irregulares apresenta um maior grau de capilaridade que estruturas isométricas [31, 32]. Modelamento de permeação de liquido em meio poroso vítreo [33] estabeleceu que a capacidade de absorção e o perfil histérico da percolação em meio vítreo poroso, como no sistema aqui apresentado, são fortemente dependentes do formato dos poros e das partículas que constituem o meio poroso. Esse grau de capilaridade pode ser inferido pela taxa de absorção, o que no caso do capilar reflete na velocidade de deslocamento do menisco (perfil de percolação). Este dado pode ser observado na Fig. 5 na qual temos a medida do deslocamento do menisco em função do tempo (para absorção de água destilada) até sua estabilização indicando que no capilar composto com a ponte porosa a absorção é maior para um mesmo tempo se comparado com o capilar único oco [25]. Embora com comportamento similares, o efeito da presença da ponte porosa é melhor visualizado se fizermos um ajuste linear na região em que ocorre absorção contínua (até aproximadamente 60 s), Fig. 6.

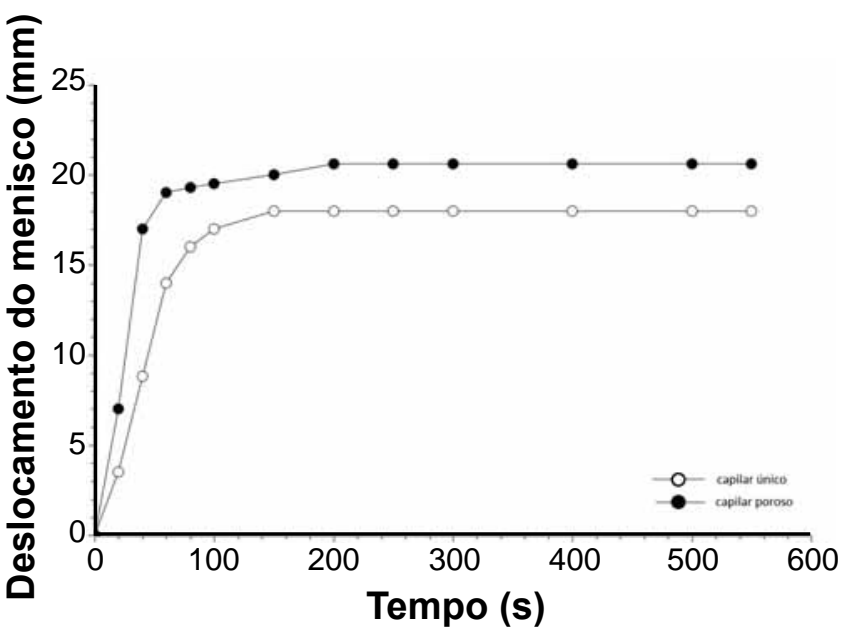

Figura 5: Perfil de percolação, representado pela velocidade de deslocamento do menisco para os sensores de capilar único e de meio poroso, indicando o favorecimento da absorção por capilaridade no meio poroso.

[Figure 5: Percolation profile, described by the velocity of meniscus movement for the tested hollow and porous medium sensors, pointing to a superior absorption due capillarity effect in the porous medium.]

Temos nestas condições que embora o capilar com ponte porosa descreva uma percolação contínua com ajuste linear inferior $\left(\mathrm{R}^{2}=0,84\right)$ do que a observada para o capilar único $\left(\mathrm{R}^{2}=0,97\right)$, a taxa de absorção, ou neste caso, a velocidade do deslocamento do menisco, pode ser estabelecido pela 
inclinação da reta (derivada $\mathrm{dy} / \mathrm{dx}$ ), indicando valores de $0,23 \mathrm{~mm} / \mathrm{s}$ para o sensor oco e de $0,35 \mathrm{~mm} / \mathrm{s}$ para o poroso sinterizado, ou seja, a presença da ponte porosa na extremidade do sensor elevada a capilaridade gerando um aumento da ordem de 1,5 vezes a velocidade de absorção. Após a estabilização, o volume de água absorvido é contudo muito similar para ambos os capilares $\left(9,07 \mathrm{~mm}^{3}\right.$ para o capilar oco e 10,03 $\mathrm{mm}^{3}$ para o com ponte porosa).

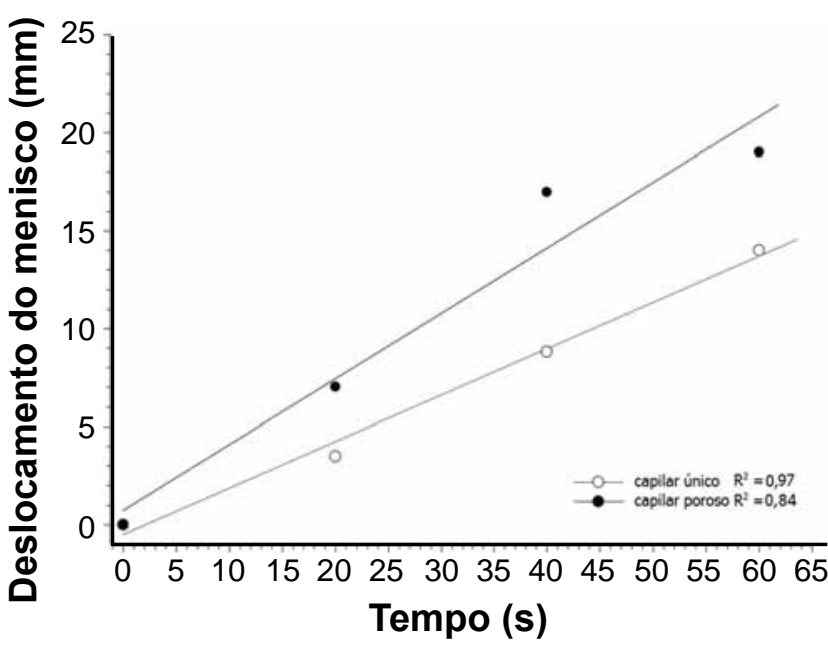

Figura 6: Detalhe da região de absorção capilar contínua, e ajuste linear.

[Figure 6: Detail of the region of continuous capillary absorption, and the linear curve adjustment.]

\section{CONCLUSÕES}

Região porosa empregada como elemento de absorção líquida, ideal para a confecção de sensores de medida de potencial mátrico, foi confeccionada à base de vidro fazendo uso da técnica de preenchimento. Essas regiões foram processadas na extremidade de capilares vítreos, empregando aquecimento de chama para a sinterização de pós de vidro a base de silicato de sódio e sal como elemento solúvel não sinterizável. Após a remoção do sal por lixiviação em água corrente, análises microscópicas indicam a consolidação entre as partículas de vidro e o estabelecimento de uma matriz de alta permeabilidade. Medidas conduzidas na absorção de água destiladas indicam melhora na velocidade de percolação com deslocamento do menisco ao longo do capilar numa taxa de 1,5 vezes superior à medida para o capilar oco.

\section{AGRADECIMENTOS}

Ao CNPq e à Embrapa (Rede AgroNano) por recursos recebidos.

\section{REFERÊNCIAS}

[1] G. W. Scherer, J. Am. Ceram. Soc. 60, 5-6 (1977) 236239.
[2] G. W. Scherer, D. L. Bachman, J. Am. Ceram. Soc. 60, 5-6 (1977) 239-243.

[3] G. W. Scherer, J. Am. Ceram. Soc. 68, 4 (1985) 216-220.

[4] J. Frenkel, J. Phys. 9 (1945) 385-391.

[5] .net/vitan-luftreiniger-vl-120.html? \&L=1>, acesso em 28/11/11.

[7] N. Marek, B. Wladyslaw, Optica Applicata 30 (2000) 613-618.

[8] K. S. Take, M. K. Kurosawa, Exhaust gas purifier for methanol-fueled engines, US Patent n. 5269140 (1993).

[9] J. Dorvee, M. J. Sailor, Phys. Status Solidi A: Appl. Res. 202 (2005) 1619-1623.

[10] C. Byung-Chul, X. Li-Hua, K. Hyung-Taek, D. W. Bahnemann, J. Ind. Eng. Chem. 12 (2006) 663-672.

[11] N. M. Bobkova, N. I. Zayats, T. V. Kolontaeva, G. N. Pun'ko, G. B. Zakharevich, Glass Ceram. 57 (2000) 412414.

[12] J. Moura, L. N. Teixeira, C. Ravagnani, O. Peitl, E. D. Zanotto, M. M. Beloti, H. Panzeri, A. L. Rosa, P. T. de Oliveira, J. Biomedical Mat. Res. Part A. 82A (2007) 545557.

[13] O. B. G. Assis, L. C. Claro, J. Non-Cryst. Solids 247 (1999) 237-240.

[14] T. Mizutani, J. Pharm. Sci. 69 (1980) 1226-1227.

[15] S. Caron, P. Bernard, M. Vernon, J. Lara, Sens. Act. B 102 (2005) 198-206.

[16] O. B. G. Assis, Cerâmica 52, 321 (2006) 105-113.

[17] R. M. Takarashi, O. B. G. Assis, Cerâmica 54, 332 (2008) 462-465.

[18] J. Caron, J. Boudreau, Soil water potential detector, US Patent 7,005,622 B2 (2006).

[19] A. Evstrapov, N. Esikova, G. Rudnitskaya, T. V. Antropova, Optica Applicata 15 (2010) 333-340.

[20] J. D. C. Pessoa, A. G. Calbo, Braz. J. Plant Physiol. 16 (2004) 17-24.

[21] V. Bertucci Neto, J. D. C. Pessoa, P. E Cruvinel, A. G. Calbo, Automação de um sistema de medida de turgescência em plantas, Pesquisa em Andamento 15 (1997) 1-3. (Publicação Interna Embrapa).

[22] J. D. C. Pessoa, A. G. Calbo, Caracterização do comportamento compressivo-expansivo de amostras fluídas com volume da ordem de micro-micro litros, in Anais I SIAGRO, S. Carlos, EMBRAPA-CNPDIA (1997) p. 411. [23] J. D. C. Pessoa, A. G. Calbo, Rev. Eng. Agríc. 12 (2001) 210-217.

[24] J. D. C. Pessoa, Instrumentações para estudo das relações água-planta, Tese, Ecologia e Recursos Naturais, UFSCar, S. Carlos, SP (1999).

[25] J. D. C. Pessoa, A. G. Calbo, Rev. Sci. Instr. 75 (2004) 2213-2215.

[26] F. B. Siebers, N. Greiulich, W. Kiefer, Glastech. Ber. 62 (1989) 63-73.

[27] Santa Marina, Catálogo Técnico (2006).

[28] D. C. Vieira, Fabricação de elementos vítreos porosos para o depósito de polímeros visando a obtenção de membranas com superfícies ativas, Tese Dr., Ciência e Engenharia de Materiais, USP, S. Carlos, SP (2001) 146p. 
[29] Y. Chiang, D. Birnie III, W. D. Kingery, "Physical Ceramics", John Wiley Sons, Inc., N. York, EUA (1977) p. 392-398.

[30] Bico de Bulsen, in http://www.infoescola.com/ materiais-de-laboratorio/bico-de-bunsen/. Acesso em 28/01/12.
[31] C. L. Prevedello, "Física dos Solos com problemas resolvidos", Curitiba, PR (1996).

[32] A. E. Scheidegger, "The Physics of Flow through Porous Media”, Univ. Toronto Press, Canadá (1960).

[33] A. Grosman, C. Ortega, Langmuir 24 (2008) 39773986.

(Rec.06/02/2012, Ac. 08/06/2012) 